\title{
Hydrodynamic shadowing effect during precipitation of dendrites in channel flow
}

\author{
Christopher Hawkins, ${ }^{1}$ Luiza Angheluta, ${ }^{1}$ and Bjørn Jamtveit ${ }^{2}$ \\ ${ }^{1}$ Physics of Geological Processes, Department of Physics, University of Oslo, 0316 Oslo, Norway \\ ${ }^{2}$ Physics of Geological Processes, Department of Geosciences, University of Oslo, 0316 Oslo, Norway \\ (Received 20 November 2013; revised manuscript received 23 January 2014; published 5 February 2014)
}

\begin{abstract}
A supersaturated fluid flowing over a reactive, rough surface leads to complex precipitation patterns. We study the growth and interaction between discrete precipitates along a reactive wall in a nonlaminar channel flow. We show that the competition between advective transport, diffusion, and mixing strongly influences the downstream precipitates morphology and the typical correlation length between different precipitates.
\end{abstract}

DOI: 10.1103/PhysRevE.89.022402 PACS number(s): 68.70.+w, 47.70.-n, 47.27.Cn, 47.54.-r

\section{INTRODUCTION}

Mineral scale formation due to the precipitation of dissolved solids is a significant problem in many fluid-bearing systems that are saturated with one or more solid components. This phenomenon may lead to clogging or a substantial decrease of transport efficiency in pipeline systems. Many industrial and domestic applications rely on pipes to transport fluids, such as oil and gas [1], renewable energy such as geothermal [2,3], chemical manufacturing, and many others. In such cases, the precipitation from a supersaturated fluid is a common phenomenon that can block the system from operating effectively. Hindering or removing the mineral scale is often a costly, time-consuming process and most often challenging to control. It would therefore be of practical value to better understand the process of precipitation under flow in pipelines in order to assess the controlling parameters for the growth conditions that may lead to pipe blockage and clogging.

Surface growth processes have been extensively studied both theoretically and experimentally, e.g., Refs. [4-7]. Nonetheless, the precipitation dynamics and pattern formation are well understood mainly in the diffusion-limited growth regime or under a laminar flow [8,9], but there are open challenges remaining as soon as the surface growth is in the presence of a nonhomogeneous and nonlaminar flow. The generic features of the precipitation patterns are rather robust with respect to changes in the detailed aqueous chemistry and interfacial composition, and are similar to the solidification patterns that are developing during growth from a supercooled liquid $[6,10]$. The similarity between generic growth morphologies during freezing and precipitation also suggests a generic deposition model that would be able to capture the universal features of these growth processes.

At the continuum level, the modeling approach to a surface growth process is based on boundary tracking methods, either in the sharp-interface formulation as in the level-set method, e.g., Ref. [11] or in the diffuse interface approximation used in the phase-field method, e.g., Refs. [12,13]. Although, the phase-field approach aims at modeling generic growth features, the solidification patterns obtained with this model share many properties similar to those observed in nature and experiments $[9,14]$. Recently a phase-field model for precipitation has been proposed as an efficient tool to study surface morphology developed by precipitation from a supersaturated solution $[5,15]$. For certain conditions of supersaturation and interfacial energy, the precipitation surface develops dendritic structures similar to those developed during solidification from an undercooled liquid in hydrostatic equilibrium [4].

To study the problem of mineral scaling in pipes with high flow rates and turbulent mixing, we couple the phase-field model for the precipitation growth with a lattice Boltzmann model for the fluid dynamics. These two models were chosen for their simplicity especially in the lattice Boltzmann models handling of complex, evolving geometries, accuracy, and compatibility (i.e., both defined on a regular grid). Here we focus particularly on the nonlaminar effects and the mixing due to the creation and shedding of vortices from the growing precipitates.

Crystal growth in the presence of laminar, shear flow has previously been shown to cause growth which leans towards the flow source, e.g., Ref. [16]. In Ref. [17] we investigated the morphology and growth orientation of an isolated precipitate growing from a supersaturated fluid with a single dissolved component at much greater flow rates and in a nonlaminar channel flow. The growth kinetics of the precipitate was assumed isotropic, i.e., without any crystallographic control on growth direction and local growth rates, and the precipitation occurs on one of the pipe's walls that has the same composition as the solute. In this paper we extend our setup to a discrete system of multiple precipitates and study the long-range interaction between precipitates and their effect on the downstream growth. This is a better approximation to pipeline systems where growth occurs in many locations upon a rough wall.

In Sec. I we motivate the paper by the field observation of precipitates in geothermal pipelines in Iceland. Good agreement is found at the generic level between our precipitation patterns and the silica scales observed in pipelines. The theoretical model for the precipitation under pipe flow is discussed in detail in Sec. II. We continue in Sec. III with the numerical approach to solving the sharp-interface model formulation from Sec. II and introduce the phase-field model coupled with the lattice Boltzmann. Some of the numerical implementation details are covered in Appendices A and B. In Sec. IV we discuss the numerical results and quantify the shadowing effect between downstream precipitates depending on dimensionless numbers as the Reynolds number, Re, and Péclet number, Pe. Concluding remarks are summarized Sec. V.

\section{FIELD OBSERVATIONS AND MOTIVATION}

Figure 1 shows an example of incipient precipitation of amorphous silica on a plate of stainless steel inserted into 
(b) (a)

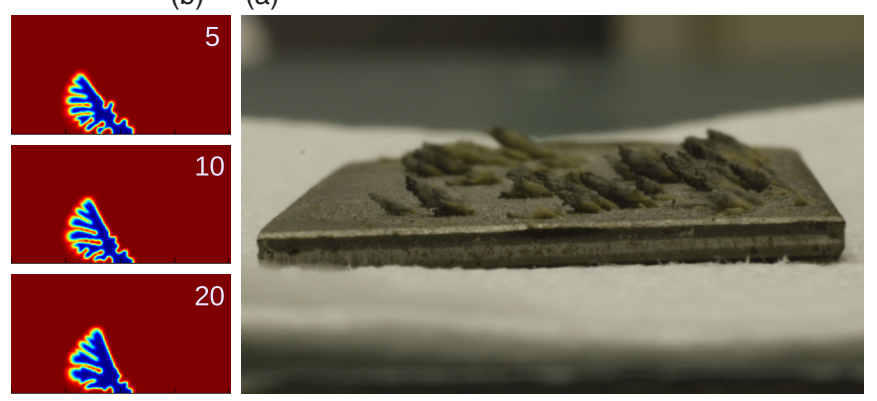

FIG. 1. (Color online) (a) Amorphous silica $\left(\mathrm{SiO}_{2}\right)$ precipitated on plates of stainless steel at the Hillisheiði geothermal power plant, Iceland. The width of the steel plate is ca. $25 \mathrm{~mm}$, and the typical length of the precipitates is $1-3 \mathrm{~mm}$. The plate was injected in the middle of a geothermal water pipe, and precipitation took place at a temperature of $120^{\circ} \mathrm{C}$ over a period of about 2 months at a flow rate around $1 \mathrm{~m} / \mathrm{s}$. (b) Numerically simulated precipitation dendrites in pipe flows at different Pe numbers. Our model captures very well the tilted growth towards the oncoming fluid and the overall shape of the precipitates, with the downstream side being more smooth while the upstream side is developing complex side branching.

the central part of a geothermal pipeline of hot water with dissolved silica at an average concentration of 800-900 ppm of $\mathrm{SiO}_{2}$ and flowing at $1 \mathrm{~ms}^{-1}$. The pipeline is about $1 \mathrm{~m}$ in diameter and located at the Hillisheði geothermal power plant, about $25 \mathrm{~km}$ east of Reykjavik, Iceland.

Two general features can be noticed from a first glance: (1) the precipitated silica structures are leaning towards the incoming flow (main flow direction is from left to right) and (2) the downstream side of each precipitate is smooth, while the upstream side tends to develop an intricate morphology. Instead of studying the particular features that may depend on the chemistry of silica precipitation or the complex, three-dimensional setup, we focus here on first understanding the generic features, such as the tilted growth and typical morphology, using an idealistic model of coupled flow and precipitation in a two-dimensional cross section of a pipeline. The right side of Fig. 1 shows few snapshots of our numerical simulated precipitation dendrites.

The samples collected from geothermal pipelines in Iceland show considerable similarity to the simulated pattern, especially at the level of generic features. It is interesting to mention that the generic growth properties of an isolated precipitate were predicted in Ref. [17] preceding the collection of sample data from geothermal pipelines. Although the results are qualitatively similar, there are some differences in the specifics, most notably the angle at which the main dendrite grows, but also the density of the upstream branching. A more detailed study that is able to capture more quantitatively the characteristics of silica precipitates is the subject of a future investigation.

\section{PRECIPITATION UNDER FLOW}

We consider that a first-order precipitation kinetics occurs at a reactive liquid-solid boundary where a single mobile solid component is transported from one phase to another. From the mass balance condition at the liquid-solid interface, it follows that the diffusive flux in the liquid near the interface equals the precipitation rate, equivalently expressed as

$$
D \mathbf{n} \cdot \nabla C=k\left(C-C_{e}\right),
$$

where $D$ is the diffusion coefficient, $C$ is the concentration of the dissolved solute, the equilibrium concentration is $C_{e}, \mathbf{n}$ is the normal to the interface pointing into the fluid, and $k$ is the constant reaction rate. The normal growth of the precipitate is described by the growth velocity $v_{n}$, which is proportional to the reaction rate as

$$
\rho_{s} v_{n}=\rho_{f} k\left(C-C_{e}\right),
$$

with $\rho_{s}$ being the mass density of the solid and $\rho_{f}$ the fluid density. Surface tension energy may also contribute to the normal growth, but, for now, we neglect surface tension effects. The solute in the fluid is diffusing and being advected by the channel flow according to the diffusion-advection equation

$$
\frac{\partial C}{\partial t}+\mathbf{v} \cdot \nabla C=D \nabla^{2} C,
$$

where the fluid velocity $\mathbf{v}$ satisfies the Navier-Stokes equations for an incompressible fluid, i.e., $\nabla \cdot \mathbf{v}=0$,

$$
\frac{\partial \mathbf{v}}{\partial t}+\mathbf{v} \cdot \nabla \mathbf{v}=-\frac{1}{\rho_{f}} \nabla p+v \nabla^{2} \mathbf{v}+\frac{1}{\rho_{f}} \mathbf{f},
$$

with $p$ being the fluid pressure field, $v$ the kinematic viscosity, and $\mathbf{f}$ the external stirring force. We assume that the solid wall is otherwise rigid and chemically inert, hence there is no diffusion or deformation in the solid phase. These equations can be rewritten in terms of the normalized concentration $c=$ $\left(C-C_{e}\right) / C_{e}$.

\section{NUMERICAL MODEL}

\section{A. The phase-field model}

Precipitation under flow is solved numerically in twodimensions using the phase-field model for surface growth [5] coupled with the lattice Boltzmann method for fluid dynamics. In the phase-field approach the liquid-solid reactive boundary is treated as a diffuse interface such that the sharp interface conditions, Eqs. (1) and (2), can be included as bulk terms into Eq. (3). A field variable $\phi$ describes the phase continuum that takes the constant values of $\phi=-1$ for a pure solid phase and $\phi=1$ for a pure liquid phase. The $\phi$ values in between are located in a narrow transition region that defines the diffuse interface; this region converges to zero in the sharp-interface limit. The dynamics of the phase field is controlled by a free energy that depends on $\phi$ and its gradient, as well as the solute concentration in the liquid phase, and it is assumed to take the following form:

$$
\mathcal{F}=\int_{V}\left[\frac{\varepsilon^{2}}{2}|\nabla \phi|^{2}+f(\phi, c)\right] d V,
$$

where $\varepsilon$ is a parameter related to the diffuse interface thickness and $f(\phi, c)$ is the local free energy density that depends linearly on the concentration field $c$ as follows:

$$
f(\phi, c)=\left(-\frac{\phi^{2}}{2}+\frac{\phi^{4}}{4}\right)+\lambda c\left(\phi-\frac{\phi^{3}}{3}\right) .
$$


The first two terms correspond to the local energy density associated with a system that allows for two equilibrium phases at $\phi=-1$ and $\phi=1$. In the absence of the other terms, these two equilibria are degenerate; i.e., they correspond to the same ground-state energy. The coupling terms to the solute concentration introduce an asymmetry in the free energy such that the solid phase is more preferable, thus mimicking the precipitation growth. $\lambda$ is a dimensionless coupling parameter. From the free energy $\mathcal{F}$, the evolution of the $\phi$ field is described by a non-Fickian diffusion along the gradient of the chemical potential $\mu=\delta \mathcal{F} / \delta \phi$ and thus is given as

$$
\tau \frac{\partial \phi}{\partial t}=\varepsilon^{2} \nabla^{2} \phi+\frac{\partial f(\phi, c)}{\partial \phi}-\varepsilon^{2} \kappa|\nabla \phi|,
$$

where the last term related to the interface curvature $\kappa=\nabla$. $(\nabla \phi /|\nabla \phi|)$ is added to remove the surface tension effects, and $\tau$ is a microscopic time parameter of the phase field $\phi$.

The evolution of the concentration field is then described by a single equation that also includes the surface precipitation condition through the $\phi$ field:

$$
\frac{\partial c}{\partial t}+\mathbf{v} \cdot \nabla c=D \nabla^{2} c+A_{1} \frac{\partial \phi}{\partial t}+A_{2} \frac{\partial \phi / \partial t}{|\nabla \phi|}\left(D \nabla^{2} \phi-\frac{\partial \phi}{\partial t}\right) .
$$

The second term on the right-hand side acts as a net source or sink of solute due to the discontinuity in the solute concentration gradient across the interface, whereas the last term corresponds to a net flux of solute arising from the discontinuity in the solute concentration across the interface. The constants in front of these terms, $A_{1}$ and $A_{2}$, are determined by ensuring that in the sharp-interface limit, $\varepsilon \rightarrow 0$, Eq. (8) reduced to the interfacial boundary conditions in Eqs. (1) and (2), which in terms of the dimensionless concentration read as

$$
v_{n}=\beta k c, \quad D \mathbf{n} \cdot \nabla c=k c,
$$

where $\beta=C_{e} \rho_{f} / \rho_{s}$. This was done in Ref. [5] in the absence of the fluid advection, and, for completeness, we redo the calculation of $A_{1}$ and $A_{2}$ and show that the fluid flow has no effect on the interfacial conditions. Equation (8) can be written in the comoving curvilinear coordinates of the interface using that $\nabla^{2} \equiv \partial^{2} / \partial n^{2}+\kappa \partial / \partial n, \nabla \equiv \partial / \partial n$, and $\partial / \partial t \equiv-v_{n} \partial / \partial n$ to arrive at

$$
\begin{aligned}
& -(\mathbf{v} \cdot \mathbf{n}) \frac{\partial c}{\partial n}+D \frac{\partial^{2} c}{\partial n^{2}}+\left(D \kappa+v_{n}\right) \frac{\partial c}{\partial n} \\
& =\left(A_{1} v_{n}+A_{2} v_{n}^{2}\right) \frac{\partial \phi}{\partial n}+A_{2} v_{n} D\left(\frac{\partial^{2} \phi}{\partial n^{2}}+\kappa \frac{\partial \phi}{\partial n}\right) .
\end{aligned}
$$

The first term on the left-hand side comes from the advection term in Eq. (8) but vanishes at the interface due to the impermeable boundary condition of the fluid dynamics, $\mathbf{v} \cdot \mathbf{n}=0$. We integrate the above equation across the interface and take the sharp interface limit in which the $\phi$ field becomes a step function with $\left.\phi\right|_{0^{-}} ^{0^{+}}=2$ and $\left.\partial_{n} \phi\right|_{0^{-}} ^{0^{+}}=0$. Thus, Eq. (10) reduces to

$$
\left.D \mathbf{n} \cdot \nabla c\right|_{0^{-}} ^{0^{+}}+\left.\left(D \kappa+v_{n}\right) c\right|_{0^{-}} ^{0^{+}}=2 v_{n} A_{1}+2 v_{n} A_{2}\left(D \kappa+v_{n}\right) .
$$

Using Eq. (9), and identifying the terms corresponding to the same powers of $v_{n}$, it follows that $A_{1}=1 /(2 \beta)$ and $A_{2}=$ $1 /(2 \beta k)$. Equation (8) can now be rewritten in a dimensionless form by rescaling space and time by typical length scale $x_{0}$ set by the diameter of the pipe and typical time scale $t_{0}$ related to the maximum inlet velocity $v_{0}$ by the relation $t_{0}=x_{0} / v_{0}$. From this rescalings, we introduce the dimensionless numbers the Péclet number, $\mathrm{Pe}=x_{0} v_{0} / D$, which measures the effect of fluid transport relative to diffusion, the Damköhler numberDa $=k x_{0} / D$, which measures precipitation rate relative to the diffusion transport rate, and the Reynolds number $\operatorname{Re}=v_{0} x_{0} / \nu$, which measures the strength turbulence in the pipe. In dimensionless units, Eq. (8) reduces to

$$
\frac{\partial c}{\partial t}=\frac{1}{\mathrm{Pe}} \nabla^{2} c+\frac{1}{2 \beta} \frac{\partial \phi}{\partial t}+\frac{\mathrm{Pe}}{2 \beta \mathrm{Da}} \frac{\partial \phi / \partial t}{|\nabla \phi|}\left(\frac{1}{\mathrm{Pe}} \nabla^{2} \phi-\frac{\partial \phi}{\partial t}\right),
$$

and the phase field Eq. (7) becomes equal to

$$
\frac{\partial \phi}{\partial t}=\frac{P_{e r}}{\mathrm{Pe}}\left[\frac{\epsilon^{2}}{x_{0}^{2}}\left(\nabla^{2} \phi-\kappa|\nabla \phi|\right)+\frac{\partial f}{\partial \phi}\right],
$$

where $P_{e r}=x_{0}^{2} /(D \tau)$ is the ratio of interface advection (due to reaction) and diffusion, and $\epsilon / x_{0}$ relates to the interface sharpness. Equations (12) and (13) are solved numerically using a finite difference scheme outlined in Appendix A.

\section{B. Lattice Boltzmann: phase-field coupling}

The lattice Boltzmann implementation [18] is detailed in Appendix B. Since the lattice Boltzmann and phase field simulations both run on regular square grids, it is straightforward to couple the two methods. In particular, the value of the $\phi$ field enters into the fluid viscosity parameter such that as $\phi \rightarrow-1$, viscosity tends to infinity; i.e., the solid phase is rigid. This can be implemented in the fluid dynamics where the Navier-Stokes equations now become

$$
\begin{aligned}
\rho_{f} & {\left[\frac{\partial f(\phi) v}{\partial t}+f(\phi) v \cdot \nabla v\right] } \\
= & -f(\phi) \nabla p+\mu \nabla^{2} f(\phi) v+M_{d},
\end{aligned}
$$

where $f(\phi)=(1+\phi) / 2$, which describes the change in the local viscosity based on the $\phi$ field. The last term on the right-hand side ensures that the velocity of the fluid is forced to zero as $\phi \rightarrow 0$, and it takes the general form

$$
M_{d}=-\frac{\nu b(1-f[\phi)]^{2} f(\phi) v}{\varepsilon^{2}} .
$$

The dimensionless constant $b$ is determined to be 2.757 by requiring that the velocity profile of the lattice Boltzmann coupled with phase-field model coincides, away from the interface and in the laminar regime, with the quadratic velocity profile of the Poiseuille flow.

In addition to the modification to the fluid viscosity it is also necessary to convert fluid cells within the lattice Boltzmann model to solid cells. This occurs when the phase value decreases below zero $(\phi<0)$. Solid cells are then subjected to the appropriate no-slip boundary conditions. 


\section{Numerical benchmarks}

We performed convergence tests of the phase-field model at a given spatial resolution and interface thickness. The convergence to the sharp-interface limit is assumed achieved when the velocity of the growing interface is unaffected by increases in resolution and interface sharpness. Further tests are run regarding the width of the interface against the size of the smallest growing dendrites. It is noted that in the case where the thickness of the interface is comparable to or greater than the smallest structures, the small-scale structures become unresolved, while the overall growth rates remain essentially the same.

The implementation of the lattice Boltzmann model is benchmarked against known solutions for pressure-driven channel flow for low and high Re numbers, and the errors are consistent with those obtained by other implementations of the same type [19]. Further tests regarding the generation of turbulent structures show qualitative agreement when compared against results generated for flow past including objects [18].

\section{RESULTS AND DISCUSSION}

\section{A. Growth structures}

We consider a two-dimensional setup of pipe flow with $O(10)$ discrete reaction sites that are randomly spaced upon one of the walls of the pipe. Since we are not modeling the nucleation of the precipitation sites, we assumed they have already been seeded and represent each of them by a half-circle of small radius where the phase field takes the value $\phi=-1$. The first 200 lattice points from the inlet are kept clear of reaction sites to ensure that no precipitate grows outside of the boundary within the time span of the simulation. The pipe walls take no primary phase value, and hence no initial growth occurs upon them. Flow in the channel is induced by a constant inlet flux where the velocity has a parabolic profile with maximum $v_{0}$ at the center of the pipe. In the absence of precipitation the velocity profile remains constant. Fluid is removed at the outlet through the open boundary conditions where the fluid velocity and concentration gradients vanish. Initially the fluid is uniformly supersaturated with a concentration field given by $c=1$ in dimensionless units. Snapshots of the precipitation structures in a pipe flow at $\mathrm{Pe}=5$ and two different $\mathrm{Re}$ numbers are shown in Fig. 2, where we notice that the shape and size of the dendrites vary heterogeneously down the pipe.

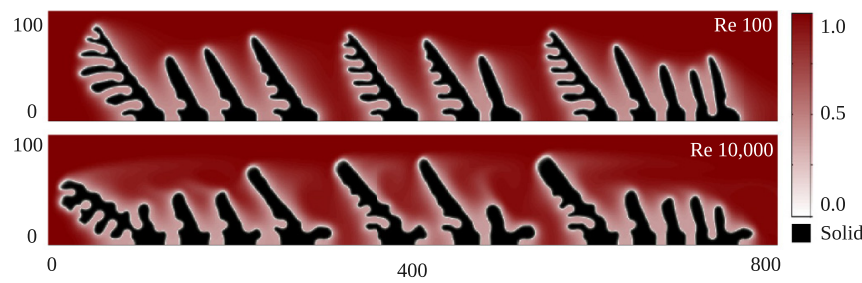

FIG. 2. (Color online) Snapshots of the growth morphology of a random array of precipitates that are growing in a supersaturated fluid flowing at two different Re numbers and at $\mathrm{Pe}=5$. The color coding corresponds to the dimensionless concentration field.

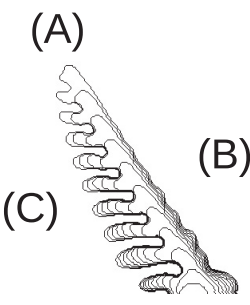

FIG. 3. Reference of labeled points corresponding to different parts of a dendrite.

The evolution and morphology of the precipitates are perhaps better explained if we differentiate between different growth regions along a selected dendrite. These key areas are labeled as (A) for the tip of the dendrite, (B) for the downstream side of the dendrite relative to the flow direction, and $(C)$ for the upstream side. These regions are also illustrated in Fig. 3 and, as we will show below, may experience different hydrodynamic environments.

In Fig. 4 we compare between different typical morphologies of a dendrite in a system of several growing dendrites for different Re and Pe numbers, where, in each case, the selected dendrite is growing from the same initial nucleation point and represents the most dominant dendrite in the system. One of the first features that we notice is that the side branching gets more suppressed with increasing $\mathrm{Re}$ number or as the flow becomes more nonlinear. Thus, the structures appear more columnar and their orientation is mainly determined by the inclination of the dendrites tips.

To better quantify the main growth direction, we calculated the growth angle of a precipitate as the average of the angles for each part of the dendrite relative to the horizontal axis. Thus, for a symmetric, normal growth, the dendrite angle is at $90^{\circ}$. This growth angle provides an estimate of the deviation from the normal growth characteristic to the diffusion-limited regime of an isolated dendrite. The main angle or asymmetry is primarily determined by the inclination at which the tip [region (A)] of the dendrite grows (this is also illustrated in Fig. 4). Thus, side branching and growth on the downstream side in region (B) act to reduce asymmetry, while the growth on the upstream side in region $(C)$ acts to increase it. Figure 5 (top) shows the main growth angle of a dendrite tip for different Pe and Re numbers. This is calculated for the selected dendrite as presented in Fig. 4. We notice that even in the diffusion-limited regime, i.e., $\mathrm{Pe} \rightarrow 0$, the main inclination of the dendrite deviates slightly from $90^{\circ}$ due to its competition with neighboring dendrites. Each precipitate affects the concentration gradient locally, and thus it influences the precipitation rate of the nearest neighbors. The deviation from the normal growth in the diffusion-limited regime is thus entirely determined by the mean separation distance between dendrites, such that in the dilute limit where the separation between dendrites is much larger than the diffusion length scale we expect the growth to be normal.

We also computed a global asymmetry angle by averaging the growth angle over all dendrites in the system excluding the one closest to the inlet, since it is not affected by any upstream structures. In Fig. 5 (bottom plot) we show the average asymmetry angle as a function of Pe and Re numbers. Both for 
$\mathrm{Pe}$

1
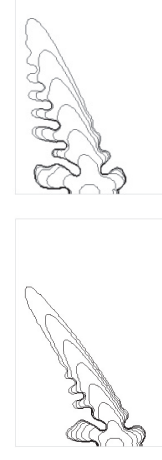

3

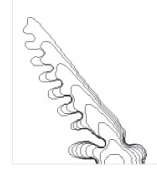

5

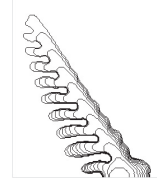

10

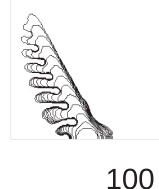

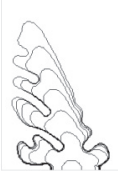
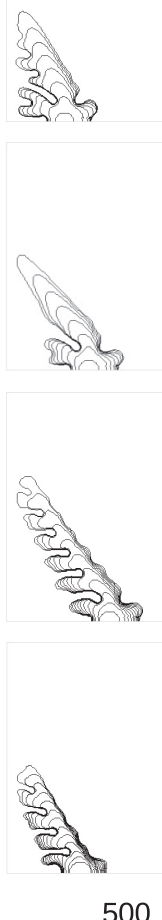
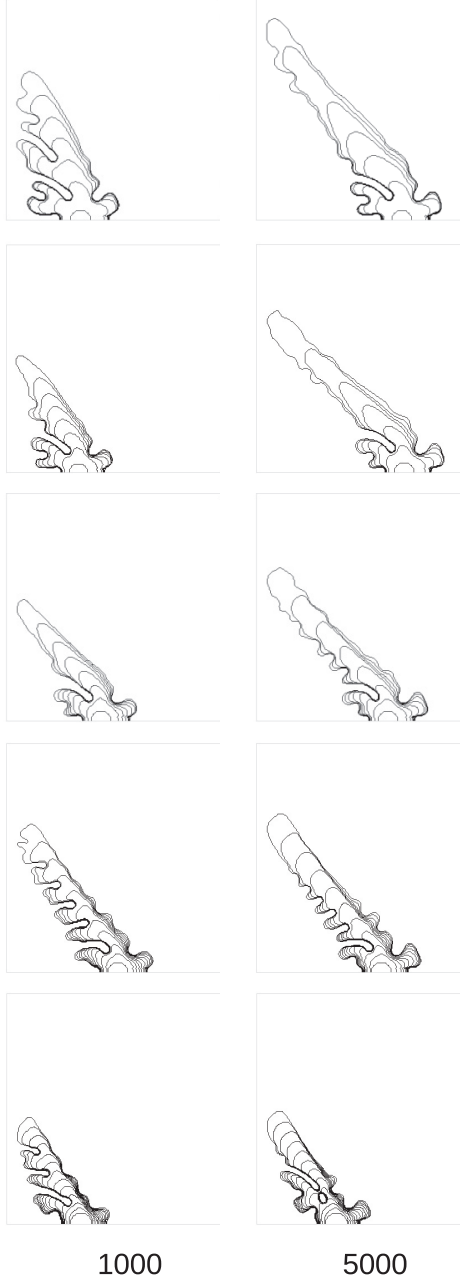
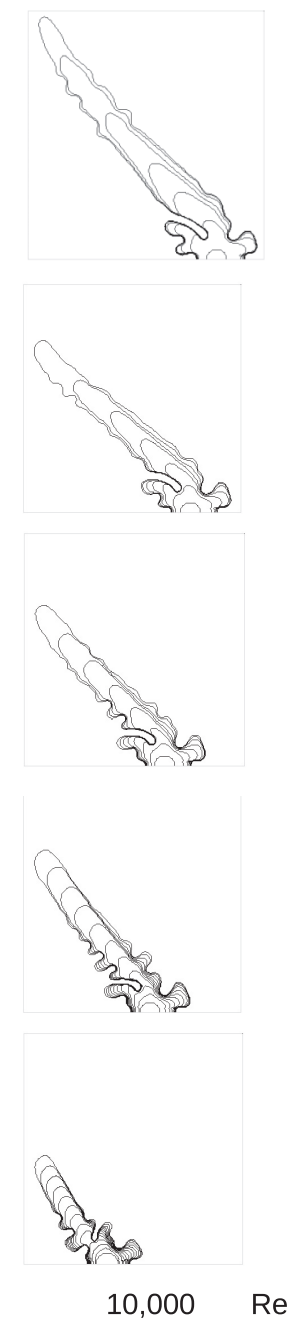

FIG. 4. Simulated evolution of the precipitation growth of a dendrite within a system of dendrites for various Pe and Re numbers. Contour lines show the position of the precipitation front at different times with time separation between $10 \mathrm{~s}$.

the tip angle and the average growth angle, the most dramatic inclination happens around $\mathrm{Pe} \sim 1$ where the diffusive and advective transport occur on similar time scales. While the tip angle keeps increasing towards $90^{\circ}$ with increasing Pe number, we notice that the average angle of the precipitates eventually becomes independent of the Pe number. Interestingly, the main inclination angle of the dendrite tip gets more oblique with increasing Re number, while the average growth angle tends to come closer to $90^{\circ}$. Because of the enhanced diffusion in region (B) due to turbulent mixing, the smooth downstream side of the dendrite becomes unstable and side branching may occur as seen in Fig. 4, which effectively brings the average growth angle towards $90^{\circ}$.

In what follows, we are going to detail the effect that different transport mechanisms, i.e., advection, diffusion, and mixing, have on the solute concentration field in the pipe, and hence on the morphology of the precipitates.

\section{B. Advection}

Growing structures that protrude into the pipe deform the fluid streamline around them such that the highest fluid velocities are present at the point extending furthest into the channel, i.e., the tip of the dendrite (A). Fluid velocities are considerably lower on the dendrite sides, i.e., regions (B) and (C) in Fig. 3; hence the local growth rates in these regions are controlled by diffusion and, at high $\mathrm{Re}$, by turbulent mixing. In region (A), the solute-depleted fluid is advected downstream, thus exposing the dendrite tip to supersaturated fluid and higher precipitation rates. The most upstream part in region (A) experiences the steepest concentration gradients, hence at low advection rates (low $\mathrm{Pe}$ ) the growth tends to also tilt steeply towards the oncoming fluid. By increasing the advective transport, a greater area of the surface in region (A) is exposed predominantly in the downstream direction; this increases not only the tilt angle but also the width of the main dendrite. This is clear in the top half of Fig. 5 where the main angle drops to its lowest point around $\mathrm{Pe} \approx 1-3$ before increasing again for higher Pe numbers. Figure 6 demonstrates this further by showing the effect which advection has upon the concentration field and hence the growth angle of the dendrite.

In Fig. 7 we show that the nonlaminar flow also has a major influence on the main precipitation angle. With increasing $\mathrm{Re}$ number, a larger area in region (A) gets exposed to higher 
(a)
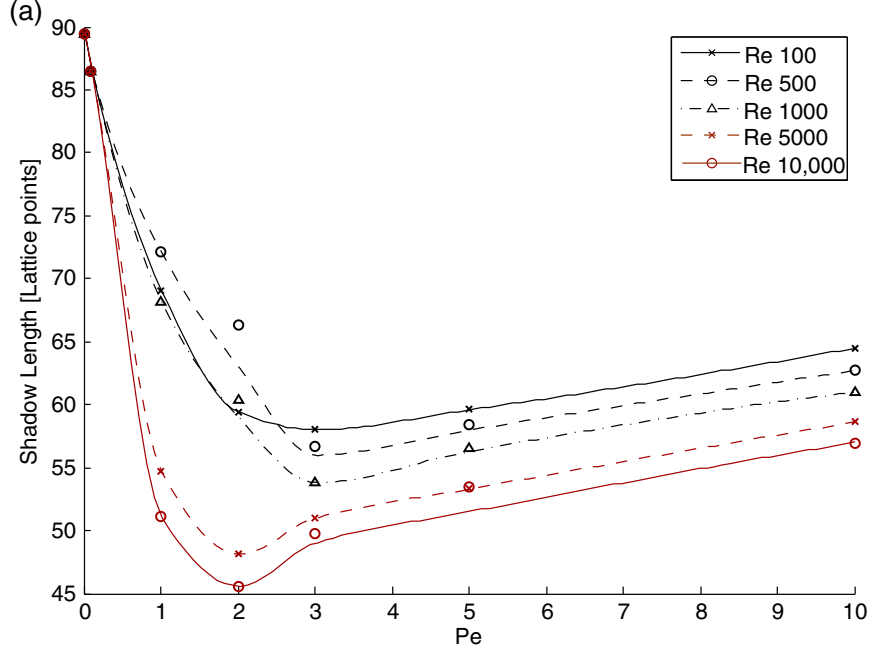

(b)

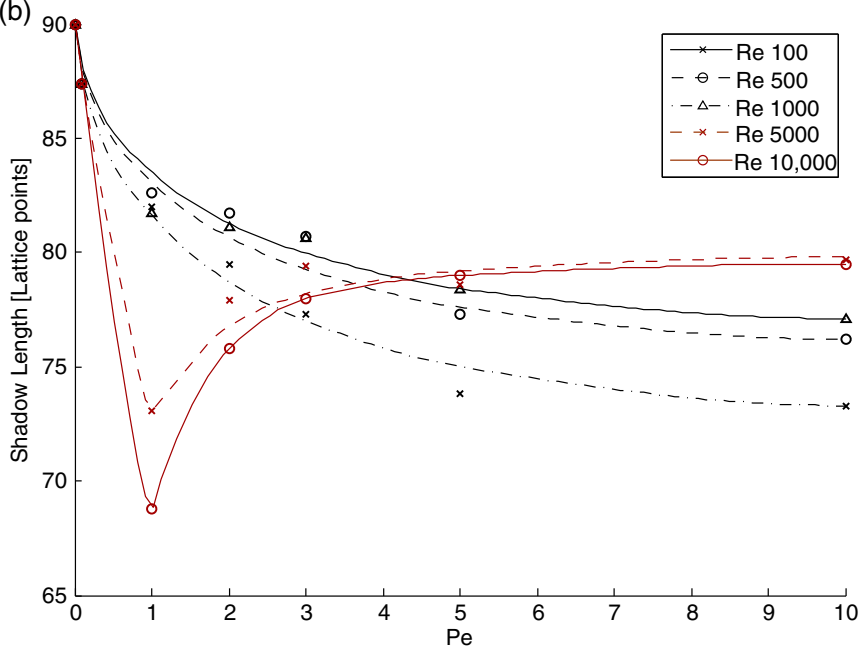

FIG. 5. (Color online) (a) The angle of tip of the growing dendrite plotted at a fixed time for different values of Pe and Re numbers. (b) The growth angle of asymmetry averaged over all dendrites and plotted at a fixed time for different values of Pe and Re numbers. The curves trace the numerical data points.

fluid velocities. Thus the solute-depleted fluid near the dendrite tip gets quickly advected and mixed with the supersaturated fluid downstream through the generation and shedding of vortices. Thus the downstream side in region (B) experiences a more mixed concentration field, and the growth becomes diffusive-like but with a faster precipitation rate since mixing enhances the effective diffusivity coefficient. Eventually the solute-depleted fluid from other upstream dendrites will also reach region $(\mathrm{C})$, depending on the fluid velocity (and therefore the Re number). At higher Re numbers, the effect of the upstream dendrites on the region $(\mathrm{C})$ of the reference dendrite will also be more pronounced resulting in the suppression of side branching on the upstream side and the formation of columnar-like structures.

\section{Diffusion}

In the diffusion-dominated region, all sides of an isolated dendrite experience a symmetric concentration field. Thus the
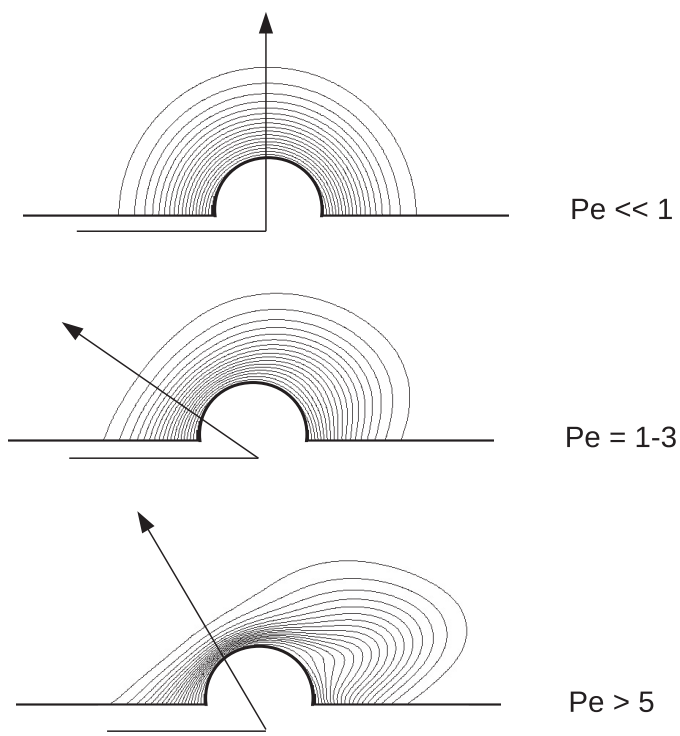

FIG. 6. Simulated advection of a diffusing source with fluid flow over a semicircular inclusion attached to a channel wall for $\operatorname{Re}=100$. Contour plots of the concentration field after it has diffused by the same distance. Arrows show the direction of steepest concentration gradient away from the surface and hence indicate the direction along which the surface grows most rapidly.

diffusive growth of a dendrite is normal, unless it is affected by the presence of other competing precipitates that change the concentration gradient around them. The dendritic or tree-like pattern of the growth results from surface instabilities and a dynamic selection mechanism of the spacing between side branches. This has been studied extensively in crystal

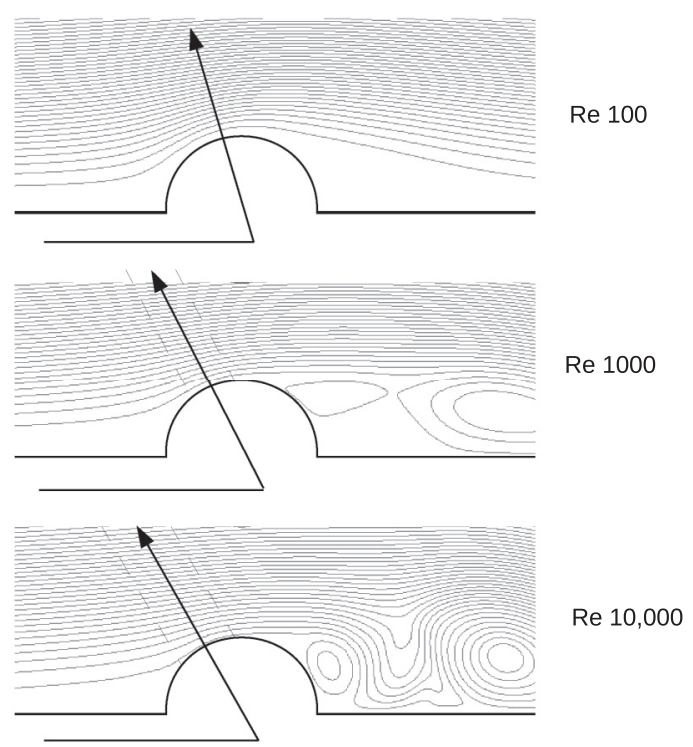

FIG. 7. Simulated flow over a semicircular inclusion attached to a channel wall for various Re numbers. Contour plots of the fluid streamlines. In each case the dotted lines indicate the area over which the fluid velocity exceeds the same defined velocity value indicating how the advection over the surface changes the growth angle and the dendrite width for different Re numbers. 
growth from undercooled liquids, e.g., Refs. [4,20]. We observe similar dendritic structures also during precipitation from a supersaturated fluid [17]. The typical length scale of growing structures is dependent upon the diffusion coefficient and the imposed mean flow. As the Pe number increases, the typical length scale reduces, and therefore finer structures are observed in regions forced to grow in a diffusion-controlled environment (B) and (C).

The concentration field is clearly lower around any evolving structure on the pipe wall, and this hinders growth in the surrounding regions. Growth will therefore be much faster in regions that are more isolated from the other preexisting structures. In our setup of a channel with randomly spaced nucleation sites, the growth will predominantly be directed away from the channel wall and towards the center of the pipe.

\section{Mixing}

Vortices formed behind growing precipitates promote efficient momentum transport across the pipe. This enables the solute-depleted fluid near the precipitation to mix faster with the supersaturated main stream fluid. In particular, mixing becomes an efficient solute transport process with increasing Re number. For an isolated precipitate growing in the presence of fluid flow, vortices will form downstream and eventually shed off and mix with the bulk flow. Thus, in region (B) the concentration field quickly becomes more uniform due to mixing than in region $(C)$ so that the downstream side is typically smooth while the upstream side that experiences a diffusive field with nonuniform gradients has a dendritic texture.

For a random array of precipitates scattered on the pipe's wall, mixing occurs predominantly in the spacing between growing dendrites (see Fig. 8 for the depiction of the standing vortices between obstacles for different Re numbers).

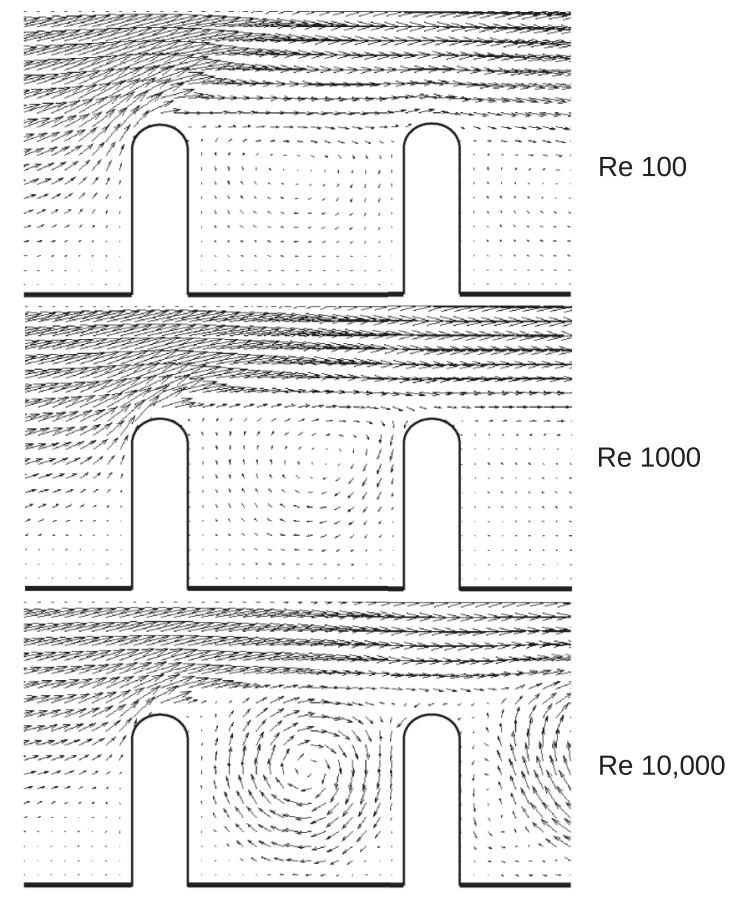

FIG. 8. Velocity vector field of a simulated flow over two simplified structures attached to a solid wall at various Re.
Therefore, both regions (B) and (C) of the selected dendrite will be affected by local mixing of the concentration field. This effect becomes more dominant with increasing Re number leading to a more even suppression of side branching in (B) and $(C)$ and hence the formation of columnar or needle-like precipitates as seen in Fig. 4.

\section{E. Shadowing length}

Pipe flow has an drastic effect on the typical interaction length scale $L_{s}$ between fast-growing dendrites. To have an estimate of how $L_{s}$ depends on Pe and Re numbers, we consider a setup where the initial asperities on the pipe's wall are spaced with an increasing distance between them. The spacing between the first two rough elements is 20 lattice points, and it increases to 40 grid points for the next two asperities, 60 grid points for the third pair, and so on. The dendrites that are almost independent of each other will have a maximum growth relative to the other dendrites that are overshadowed. We measure the minimum distance between these fast-growing dendrites as a proxy of the shadowing length. We also run a second set of simulations for each Pe and Re numbers, where the initial distance between asperities decreases downstream. This provides a better estimate of the shadowing length scale obtained as the average of the minimum distance calculated from the two protocols.

Figure 9(a) shows the shadowing length $L_{s}$ as a function of Pe and Re. Large $L_{s}$ means a longer-range interaction between fast-growing dendrites and the presence of more overshadowed

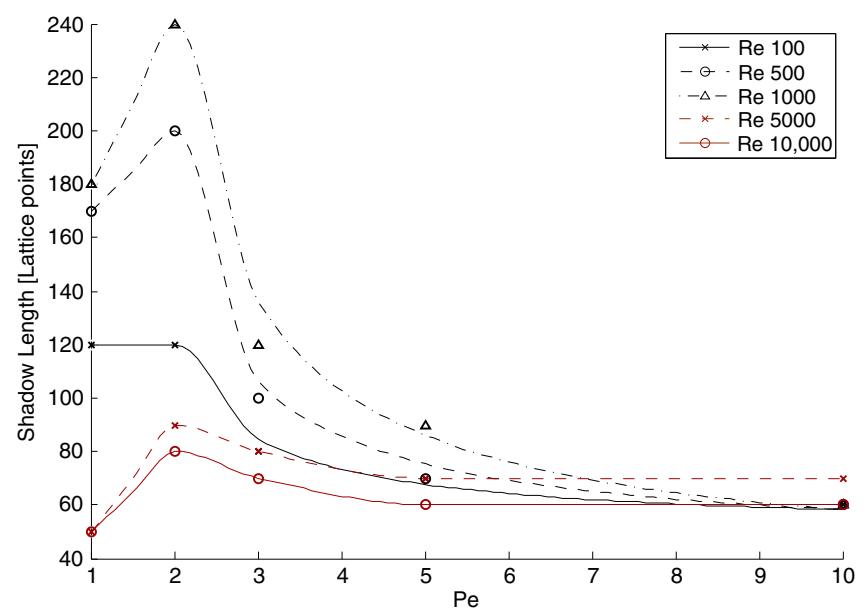

(a)

(b)

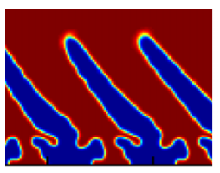

80

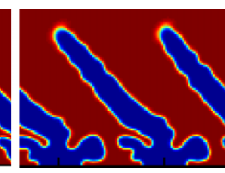

100

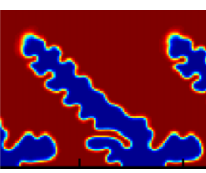

140

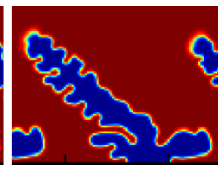

160
FIG. 9. (Color online) (a) Plot of the minimum distance between fast growing dendrites for different values of Pe and Re numbers. (b) Simulations of equally spaced nucleation sites with spacing $(L=$ $\{80,100,140,160\})$ above and below the observed shadowing length calculated at $L_{s} \approx 120$, for $\mathrm{Re}=100$ and $\mathrm{Pe}=2$. 


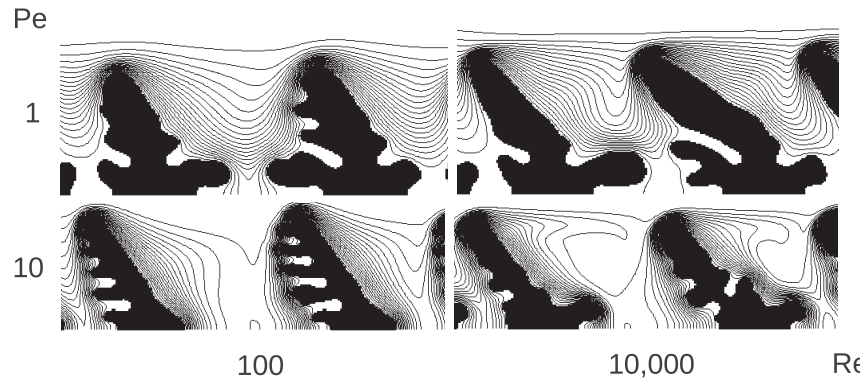

FIG. 10. Contour plot of the concentration field around two precipitation dendrites growing in the presence of other dendrites in the pipe for different values of Pe and Re numbers. The main flow is from the left to the right.

dendrites in between. Slow-growing dendrites evolve in a calmer environment with less fluid streaming. This causes them to grow more diffusive-like, but their main orientation is still dictated by fast-growing structures around them. The growth direction of the slow dendrites is typically along the path where no other growing structures are present. To eliminate the possible effects of a nonuniform spacing between precipitates, we have also run simulations of equally spaced dendrites where the spacing takes values slightly above and slightly below the calculated shadow length $L_{s}$ from Fig. 9(a). An example of this is shown in Fig. 9(b) for $\mathrm{Re}=100$ and $\mathrm{Pe}=2$, where a clear difference can be seen for spacing above and below the shadow length of $L_{s} \approx 120$.

Figure 10 shows the concentration field around two neighboring dendrities for several Pe and Re numbers. The contours of the concentration field provide an illustration of the shadowing effect that takes place where the concentration field affects the growth rate of dendrites in the downstream direction. The shadowing length depends both on the diffusion and advection/mixing length scales, and it peaks when these two typical lengths are of the same order of magnitude, i.e., $\mathrm{Pe} \approx 1$ as seen in Fig. 9. As the simulation is set up to run on the advective time scale, varying the Pe number is equivalent to changing the rate of diffusion while keeping advection constant for a given Re number. Hence increasing the Pe number reduces the shadowing length. Larger values of $\mathrm{Re}$ number enhance the advection rate at the interface, therefore increasing the advective length and hence the shadow length.

As the Re number increases the vortex which forms in between two neiboring dendrites increases in size and magnitude (also shown in Fig. 8). At high Re and Pe numbers (where mixing is the dominant transport mechanism) the vortex is able to effectively trap solute-depleted fluid flowing downstream stopping it from reaching region (A). This allows the tips to grow more freely at closer distances causing an apparent shorter shadowing length.

\section{F. Growth rate}

In addition to growth morphology, the overall growth rate also varies significantly as seen from Fig. 4; most notably the rate decreases for increasing Pe number and for the most part increases with increasing Re number.

The dependence of the growth rate on Pe number can be determined (see Appendix C) for a flat interface in the laminar regime, in which case $v_{n} \propto 1 / \mathrm{Pe}$. The inverse proportionality of the interface velocity with the Pe number is consistent with the growth rates observed from Fig. 4. The effect of the Re number on the growth velocity is harder to quantity. Overall, our numerical simulations suggest that increasing the advection rate at the solid-liquid interface acts to increase the growth rate of the system. However, it is also apparent that the growth rate initially decreases with increasing Re number. For a fixed $\mathrm{Pe}$, a minimum growth rate is observed around $\mathrm{Re} \approx 500-1000$. From Fig. 9, we notice that this slow growth regime corresponds to the largest shadowing length.

\section{CONCLUSIONS}

This paper studies the effects of interaction between growing precipitates in a channel flow at varying flow rates. Three mechanisms work in competition to determine the morphology of the dendrites which develop on one of the pipe's reactive walls. Diffusion acts equally in all areas to produce largely isotropic dendritic structures with a typical finger width that decreases with decreasing diffusion rate. Advective transport promotes an increase in the solute concentration at points that protrude into the channel by removing solute-depleted fluid further downstream, and thus accelerating the growth against the mainstream flow direction. Shallowest growth angles (the most directed towards oncoming flow) occur at low advection rates where only the most upstream regions affected by advection are introduced to supersaturated fluid. With increasing flow rates, the regions further downstream are also exposed to the incoming fluid, and this increases the finger's width and the growth angle of the dendrites. Increased Re effectively amounts to a more asymmetric growth towards the incoming fluid and a wider width of the main dendritic branch. Solute-depleted fluid is transported downstream where it hinders precipitation growth, and the size of the affected area is dependent upon the rate of advection relative to the growth time scale. Enhanced mixing occurs in the presence of vortices shed downstream from the growing dendrites. The interaction between eddies promotes momentum transport across the pipe diameter and thus mixes the solute concentration much faster than by molecular diffusion. Mixing is dependent upon Re number and acts faster with increasing Re. The effect of mixing on the precipitation growth is such that it induces a more diffusive-like growth of the precipitates but much larger precipitation rates due to larger effective diffusivity.

The typical shadowing length between interacting dendrities in pipe flow is controlled by the combination of diffusion and advection length scales on the time scale of growth. In the laminar regime, an increase in diffusive or advective length scales will correspond to an increase in shadowing length. At higher Re numbers, however, the flow pattern and increased circulation velocities between dendrites force depleted fluid away from the tip, reducing the length between dominant dendrites.

\section{ACKNOWLEDGMENTS}

We are grateful to Ingvi Gunnarsson and collaborators at Reykjavik Energy who provided access to silica scales grown on thin plates inserted in geothermal pipelines at the 
Hellisheidi Power Plant. One image of these silica scales is shown in Fig. 1. We also benefitted from discussions with Liane Benning and Daniela Meier, University of Leeds. This study was supported by the Center for Physics of Geological Processes and a fellowship from MINSC, a European Marie Curie Initial Training Network.

\section{APPENDIX A: FINITE DIFFERENCE}

Equations (12) and (13) are solved by an isotropic finite difference method where nearest and next-nearest neighbors are considered. The partial derivatives and Laplacian operator are discretized as

$$
\begin{gathered}
\frac{\partial \phi_{i, j}}{\partial x}=\frac{1}{8 d s}\left(\phi_{i+1, j+1}+2 \phi_{i+1, j}-\phi_{i-1, j-1}\right. \\
\left.-\phi_{i-1, j+1}-2 \phi_{i-1, j}+\phi_{i+1, j-1}\right), \quad \text { (A1) } \\
\frac{\partial \phi_{i, j}=}{\partial y}=\frac{1}{8 d s}\left(\phi_{i+1, j+1}+2 \phi_{i, j+1}-\phi_{i-1, j-1}\right. \\
\left.\quad-\phi_{i-1, j+1}-2 \phi_{i, j-1}+\phi_{i+1, j-1}\right), \\
\nabla^{2} \phi_{i, j}=\frac{1}{2 d s^{2}}\left(\phi_{i+1, j}+\phi_{i-1, j}+\phi_{i, j+1}+\phi_{i, j-1}\right)-\frac{3 \phi_{i, j}}{d s^{2}} \\
+\frac{1}{4 d s^{2}}\left(\phi_{i+1, j+1}+\phi_{i-1, j-1}+\phi_{i-1, j+1}+\phi_{i+1, j-1}\right),
\end{gathered}
$$

where $d s$ is the lattice spacing, and $(i, j)$ represents the $(x, y)$ position in a discrete rectangular lattice. The equations are then propagated in time using an explicit middle point discretization,

$$
f(t+\Delta t)=\frac{\Delta t}{2}[f(t-\Delta t)+f(t)],
$$

with $\Delta t$ being the time step of the simulation.

\section{APPENDIX B: LATTICE BOLTZMANN MODEL}

The lattice Boltzmann model (for more details see Ref. [18]) solves the Navier-Stokes equations on a regular grid by combining the properties of mass, momentum, and energy conservation with an isotropic relaxation of the stress. The method is based on the idea that the fluid can be represented by a cloud of free moving particles ("momentum carriers") which occasionally interact leading to energy and momentum exchange. These particles are restricted to move at a constant speed along a discrete set of specific directions only. For two-dimensional simulations, nine directions of travel with unit vectors $\mathbf{e}_{i}$, for $i=0, \ldots, 8$, create a regular square lattice grid; this is also the base grid type for most other numerical techniques [18]. The vector labels $i$ are numbered (anticlockwise) in Fig. 11.

Additionally, fluid particles are grouped together to drastically reduce the quantity of data and numerical computations. This is possible since at any grid point there is a large number of particles traveling along the same paths with the same speed. Therefore, one can define a set of particle density functions $n_{i}$ with $i=0, \ldots, 8$ corresponding to each direction of motion.

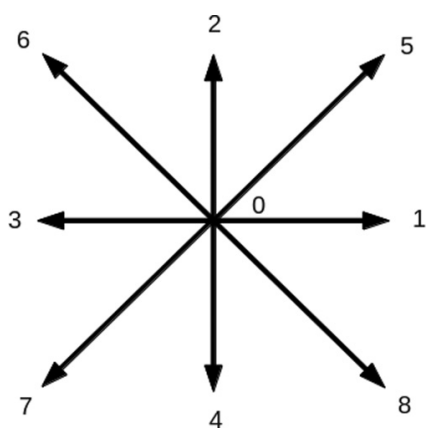

FIG. 11. Lattice representation of the discrete orientations. A $d 2 q 9$ lattice is used (two dimensions, nine directions); $i=0$ represents particles at rest.

The final component is the interaction of particles. In the case of the lattice Boltzmann model, due to restrictions of direction and speed, particles are simply scattered from one direction to another or rather from one cloud of particles to another. The evolution of the particle functions by relaxation proceeds as

$$
n_{i}\left(\mathbf{x}_{a}+\mathbf{c}_{i a}, t+\Delta t\right)=n_{i}\left(\mathbf{x}_{a}, t\right)-\frac{1}{\tau}\left[n_{i}\left(\mathbf{x}_{a}, t\right)-n_{i}^{e q}\left(\mathbf{x}_{a}, t\right)\right]
$$

where $a=1,2$ for $x, y$ respectively, $c$ is the lattice sounds speed, and $t$ is the time and $\Delta t$ the time step. The second term describes the particle interactions via collisions which push the system towards equilibrium. Here the relaxation time $\tau$ is related to the fluid viscosity as

$$
\tau=\frac{6 v+1}{2} \frac{\Delta x^{2}}{\Delta t}
$$

$v$ being the viscosity and $\Delta x$ the grid spacing. The local equilibrium distribution $n_{i}^{e q}$ to second order is

$$
n_{i}^{e q}=w_{i} \rho\left(1+\frac{3 \mathbf{e}_{i a} \mathbf{u}_{a}}{c^{2}}+\frac{9 \mathbf{e}_{i a} \mathbf{e}_{i b} \mathbf{u}_{a} \mathbf{u}_{b}}{2 c^{4}}-\frac{3 \mathbf{u}_{a} \mathbf{u}_{a}}{2 c^{2}}\right),
$$

where $u$ is the velocity, $a$ and $b$ take the values 1,2 representing $x, y$ as before, and summation is implied over indices $a$ and $b$. The weights $w_{i}$ are

$$
w_{0}=\frac{4}{9}, \quad w_{1,2,3,4}=\frac{1}{9}, \quad w_{5,6,7,8}=\frac{1}{36} .
$$

At each grid point the total mass and velocity of the fluid can be inferred by summing the contributions from each particle function. The velocity $u$ and the density $\rho$ are hence calculated as

$$
\rho=\sum_{i} n_{i}, \quad \mathbf{u}=\frac{1}{\rho} \sum_{i} n_{i} \mathbf{e}_{i} .
$$

The lattice Boltzmann model proceeds through the following stages. First, a uniform grid is set up with particle density functions moving along each of the chosen directions. At each time step, the functions move along their respective directions of travel to the adjacent lattice point. The equilibrium functions at each lattice point is then calculated, and the system is relaxed towards equilibrium. While one may (correctly) assume that on a microscopic scale the method may not be an accurate representation of a true fluid, the lattice Boltzmann method is able to capture the macroscopic details of fluids with great accuracy. 


\section{Boundary conditions}

No-slip boundary conditions are used on the wall of the pipe and for the solid interface. These are implemented within the lattice Boltzmann model using bounce-back boundary conditions; i.e., any fluid particle density functions entering the solid phase are simply reversed in direction (hence canceling out the velocity component in that direction).

For the pipe's inlet, we set a constant fluid velocity profile, while at the outlet open boundaries are implemented.

\section{Incompressible lattice Boltzmann model}

Various incompressible modifications to the lattice Boltzmann model have been derived to create a more constant density field. We use the method developed by Zou et al. [21]. Fluid velocity is assumed to be independent of the density and equal to

$$
\mathbf{u}=\sum_{i} n_{i} \mathbf{e}_{i}
$$

to ensure that the velocity field remains divergence free.

\section{Turbulence}

Simulating turbulent fluid dynamics at every scale is computationally impractical [22]. Instead we seek to model the resolved scales of turbulent motion. Scales below these are taken into account through a subgrid model which filters out the small-scale motion via a renormalization of the local viscosity. The effective viscosity becomes

$$
v=v_{0}+v_{t}
$$

Here $v_{0}$ is the fluid viscosity and $v_{t}$ the modeled eddy viscosity. We use the Smagorinsky model [23] to determine the small unresolved scale motion. Here the eddy viscosity $v_{t}$ is calculated from the filtered rate of strain tensor $S_{i, j}=$ $\left(\partial_{j} u_{i}+\partial_{i} u_{j}\right) / 2$ :

$$
v_{t}=\left(C_{s} \Delta\right)^{2} S, \quad S=\sqrt{2 \sum_{i, j} S_{i, j} S_{i, j}},
$$

where $\Delta$ is the filter width and $C_{s}$ is the Smagorinsky constant. The total modified relaxation time can now be written as

$$
\tau=\frac{1}{2} \sqrt{\tau_{0}^{2}+\frac{18 \sqrt{2}}{\rho_{0}} C_{s}^{2} \Delta S}+\tau_{0} .
$$

In the lattice Boltzmann model, the rate of strain tensor can be calculated from the local nonequilibrium particle densities and given as

$$
S_{a, b}=\sum_{i} \mathbf{e}_{i a} \mathbf{e}_{i b}\left(n_{i}-n_{i}^{e q}\right) .
$$

\section{APPENDIX C: GROWTH VELOCITY OF A FLAT INTERFACE}

An analytic solution of the precipitation growth velocity $v_{n}$ can be obtained for a laminar flow, $\mathbf{v}=\left(v_{x}, 0\right)$, near a flat interface [5]. In the steady-state and comoving frame of the interface propagating with a constant velocity $v_{n}$, the evolution equation of the concentration field reads as

$$
\frac{1}{\mathrm{Pe}} \frac{d^{2} c}{d y^{2}}+v_{n} \frac{d c}{d y}=0,
$$

where $c(y)$ is the steady-state concentration field $[c=(C-$ $\left.C_{e}\right) / C_{e}$ ] away from the interface, assuming that it is homogeneous along the interface in the $x$ direction. The general solution of the above equation follows:

$$
c=c_{1}+c_{2} e^{-v_{n} \text { Pe } y},
$$

where the integration constants are determined from the boundary conditions. We use the mass continuity at the interface $c^{\prime}(y)=$ Da $c(y)$, at $y=0$, and the far field condition $c(y)=c_{\infty}$ as $y \rightarrow \infty$. Thus,

$$
c=c_{\infty}-\frac{\mathrm{Da} c_{\infty}}{\mathrm{Da}+v_{n} \mathrm{Pe}} e^{-v_{n} \mathrm{Pe} y} .
$$

The normal growth velocity follows from interfacial boundary condition $\operatorname{Pe} v_{n}=\beta \mathrm{Da} c(y)$ at $y=0$ and is equal to

$$
v_{n}=\frac{\operatorname{Da}\left(\beta c_{\infty}-1\right)}{\operatorname{Pe}} \text {. }
$$

[1] S. Nešić, Corrosion Sci. 49, 4308 (2007).

[2] I. Gunnarsson and S. Arnórsson, Geochim. Cosmochim. Acta 64, 2295 (2000).

[3] I. Gunnarsson and S. Arnórsson, Geothermics 34, 320 (2005).

[4] J. S. Langer, Rev. Mod. Phys. 52, 1 (1980).

[5] Z. Xu and P. Meakin, J. Chem. Phys. 129, 014705 (2008).

[6] P. Meakin and B. Jamtveit, Proc. R. Soc. A: Math. Phys. Eng. Sci. 466, 659 (2010).

[7] N. Goldenfeld, P. Y. Chan, and J. Veysey, Phys. Rev. Lett. 96, 254501 (2006).
[8] J.-H. Jeong, N. Goldenfeld, and J. A. Dantzig, Phys. Rev. E 64, 041602 (2001)

[9] J. Wang, W. Zhai, K. Jin, and C. Chen, Mater. Lett. 65, 2448 (2011).

[10] B. Jamtveit and Ø. Hammer, Geochem. Persp. 1, 341 (2012).

[11] S. Chen, B. Merriman, S. Osher, and P. Smereka, J. Comput. Phys. 135, 8 (1997).

[12] A. Karma and W.-J. Rappel, Phys. Rev. E 53, R3017 (1996).

[13] W. Boettinger, J. Warren, C. Beckermann, and A. Karma, Annu. Rev. Mater. Res. 32, 163 (2002). 
[14] R. Trivedi and K. Somboonsuk, Acta Metall. 33, 1061 (1985).

[15] Z. Xu and P. Meakin, J. Chem. Phys. 134, 044137 (2011).

[16] R. Tönhardt and G. Amberg, J. Cryst. Growth 194, 406 (1998).

[17] C. Hawkins, L. Angheluta, Ø. Hammer, and B. Jamtveit, Europhys. Lett. 102, 54001 (2013).

[18] S. Succi, The Lattice Boltzmann Equation (Oxford University Press, Oxford, 2001).
[19] C.-F. Ho, C. Chang, K.-H. Lin, and C.-A. Lin, CMES: Computer Modeling in Engineering \& Sciences 44, 137 (2009).

[20] R. Kobayashi, Physica D 63, 410 (1993).

[21] Q. Zou, S. Hou, S. Chen, and G. D. Doolen, J. Stat. Phys. 81, 35 (1995).

[22] C. K. Aidun and J. R. Clausen, Annu. Rev. Fluid Mech. 42, 439 (2010).

[23] E. Lévêque, F. Toschi, L. Shao, and J.-P. Bertoglio, J. Fluid Mech. 570, 491 (2007). 